\title{
Quasiparticle-continuum level repulsion in a quantum magnet
}

\author{
K. W. Plumb ${ }^{1}$, Kyusung Hwang', Y. Qiu' ${ }^{2,3}$, Leland W. Harriger'2, G. E. Granroth ${ }^{4}$, \\ Alexander I. Kolesnikov' ${ }^{5}$, G. J. Shu ${ }^{6}$, F. C. Chou ${ }^{6}$, Ch. Rüegg, ${ }^{7,8}$, Yong Baek Kim ${ }^{1,9}$ and Young-June Kim ${ }^{1 \star}$
}

\begin{abstract}
When the energy eigenvalues of two coupled quantum states approach each other in a certain parameter space, their energy levels repel each other and level crossing is avoided'. Such level repulsion, or avoided level crossing, is commonly used to describe the dispersion relation of quasiparticles in solids ${ }^{2}$. However, little is known about the level repulsion when more than two quasiparticles are present; for example, in a strongly interacting quantum system where a quasiparticle can spontaneously decay into a many-particle continuum $^{3-5}$. Here we show that even in this case level repulsion exists between a long-lived quasiparticle state and a continuum. In our fine-resolution neutron spectroscopy study of magnetic quasiparticles in the frustrated quantum magnet $\mathrm{BiCu}_{2} \mathrm{PO}_{6}$, we observe a renormalization of the quasiparticle dispersion relation due to the presence of the continuum of multi-quasiparticle states.
\end{abstract}

A fundamental concept in condensed matter physics is the idea that strongly interacting atomic systems can be treated as a collection of weakly interacting and long-lived quasiparticles. Within a quasiparticle picture, complex collective excited states in a many-body system are described in terms of effective elementary excitations. The quanta of these excitations carry a definite momentum and energy, and are termed quasiparticles. Magnetic insulators containing localized $S=1 / 2$ magnetic moments and having valence-bond solid ground states are ideal systems in which to study bosonic quasiparticles in an interacting quantum many-body system ${ }^{6}$. The elementary magnetic excitations in these materials are triply degenerate $S=1$ quasiparticles called triplons, and their momentum- and energy-resolved dynamics can be probed directly though inelastic neutron scattering (INS) measurements.

In particular, when the system's Hamiltonian has an interaction term coupling single-particle and multi-particle states, the single quasiparticles may decay into the continuum of multi-particle states $^{3,4}$. In such a system, the Hamiltonian for the single quasiparticles is non-Hermitian and the energy eigenvalues are in general complex. The single-particle decay typically occurs in two ways. Often the single-particle mode stays as a resonance inside the continuum, but the lifetime becomes short and the mode is highly damped ${ }^{3}$. Sometimes the single quasiparticle simply ceases to exist, and the dispersion abruptly terminates when it crosses the continuum boundary ${ }^{5}$. However, there is a third possibility, in which the single-quasiparticle dispersion is significantly renormalized to avoid the multi-particle continuum. This is analogous to the wellknown avoided level crossing behaviour of coupled modes, but in the complex plane of energy eigenvalues ${ }^{7}$.

Despite broad interest in strongly interacting quantum systems, experimentally realizing an ideal condition to study the interaction between a quasiparticle and a multi-particle continuum turns out to be extremely difficult. One realization occurs in semiconducting quantum dots, where quantum interference effects between a local bound state and a continuum (Fano resonance) produce physics similar to quasiparticles in a continuum ${ }^{8,9}$. Here we show that the quantum magnet $\mathrm{BiCu}_{2} \mathrm{PO}_{6}$ presents a rare model system that allows one to study quasiparticle level repulsion in the complex plane. This phenomenon arises owing to the presence of the very large anisotropic exchange interactions in $\mathrm{BiCu}_{2} \mathrm{PO}_{6}$, originating from spin-orbit coupling. These anisotropic interactions play a dual role in $\mathrm{BiCu}_{2} \mathrm{PO}_{6}$. First, they break the degeneracy of the triplet excitations. Second, the anisotropic exchange interaction is responsible for a strong anharmonic term in the magnetic Hamiltonian which couples the single-triplon and multi-triplon excitations. As a result, the triplon lifetime may be reduced in the region of phase space where the (single) triplon dispersion overlaps with multi-triplon continuum. This decay process may in fact be so strong that the quasiparticle description ceases to be valid within the continuum. Both types of decay processes are observed in $\mathrm{BiCu}_{2} \mathrm{PO}_{6}$. Furthermore, we observe that the dispersion relation of the triplons is strongly renormalized near the boundary of the multi-triplon continuum owing to the level repulsion between the single quasiparticle and the continuum.

In the following, we first present INS measurements of the full triplon dispersion in $\mathrm{BiCu}_{2} \mathrm{PO}_{6}$, which reveal a rich excitation spectrum including a multi-triplon scattering continuum. Analysis of the triplon excitation spectrum using bond-operator theory enables us to determine the magnetic Hamiltonian of $\mathrm{BiCu}_{2} \mathrm{PO}_{6}$ accurately. We find that strong spin-orbit coupling plays an essential role in this compound through substantial symmetric and antisymmetric anisotropic interactions. We will finally discuss the interaction of the triplon quasiparticles with the continuum, which manifests as a drastic renormalization of the quasiparticle spectra and ultimately spontaneous quasiparticle decay.

\footnotetext{
${ }^{1}$ Department of Physics and Center for Quantum Materials, University of Toronto, Toronto, Ontario M5S 1A7, Canada. ${ }^{2}$ NIST Center for Neutron Research, National Institute of Standards and Technology, Gaithersburg, Maryland 20899, USA. ${ }^{3}$ Department of Materials Science and Engineering, University of Maryland, College Park, Maryland 20742, USA. ${ }^{4}$ Neutron Data Analysis and Visualization Division, Oak Ridge National Laboratory, Oak Ridge, Tennessee 37831, USA. ${ }^{5}$ Chemical and Engineering Materials Division, Oak Ridge National Laboratory, Oak Ridge, Tennessee 37831, USA. ${ }^{6}$ Center for Condensed Matter Sciences, National Taiwan University, Taipei 10617, Taiwan. ${ }^{7}$ Laboratory for Neutron Scattering and Imaging, Paul Scherrer Institute, $\mathrm{CH}-5232$ Villigen, Switzerland. ${ }^{8}$ Department of Quantum Matter Physics, University of Geneva, $\mathrm{CH}-1211$ Geneva, Switzerland. ${ }^{9} \mathrm{Canadian}$ Institute for Advanced Research/Quantum Materials Program, Toronto, Ontario MSG 1Z8, Canada. *e-mail: yjkim@physics.utoronto.ca
} 
a

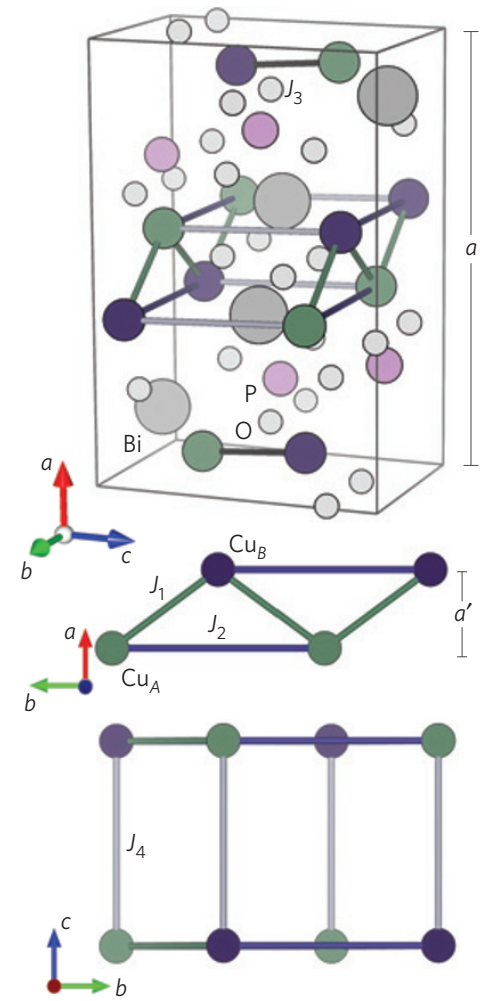

b

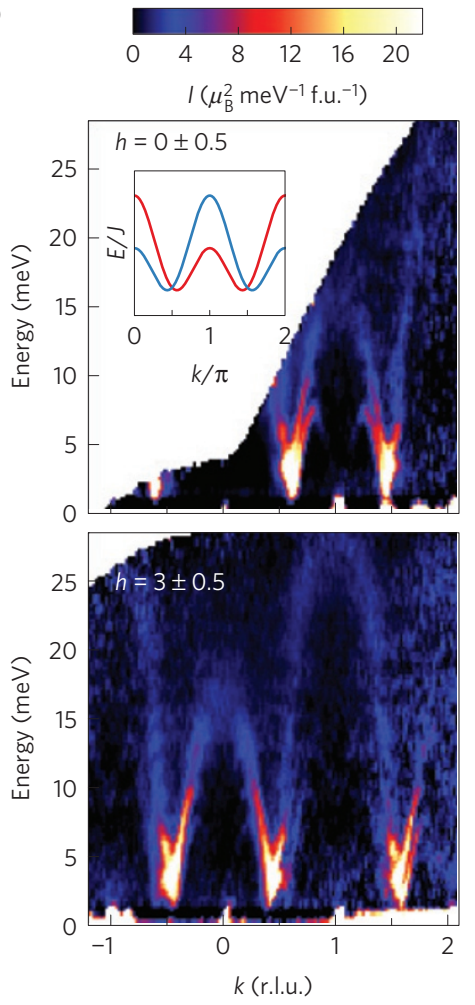

c

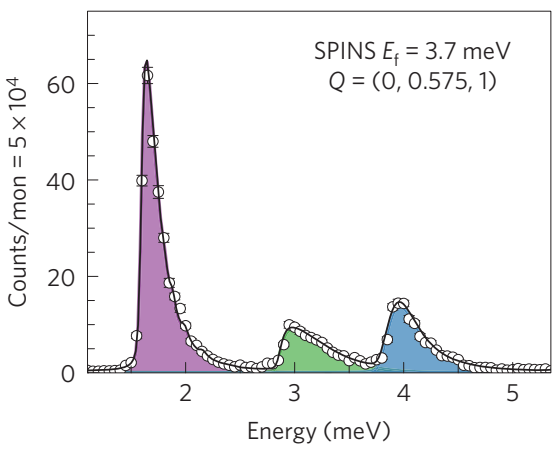

d

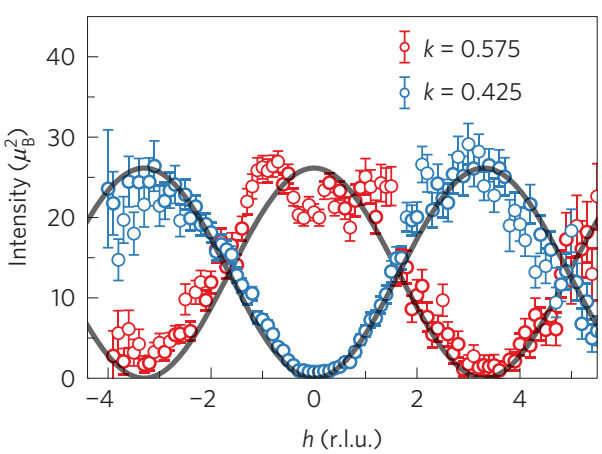

Figure 1 | Crystal structure and magnetic excitations in $\mathrm{BiCu}_{2} \mathrm{PO}_{6}$. a, The orthorhombic crystal structure of $\mathrm{BiCu}_{2} \mathrm{PO}_{6}$ contains zigzag chains of $\mathrm{Cu}^{2+}$ ions running parallel to the $b$-axis. The coupled chains can be viewed as bi-layers of $\mathrm{Cu}^{2+}$ with spacing $a^{\prime}=0.162 a$. Along the chains, neighbouring $\mathrm{Cu}^{2+}$ interact via competing nearest-neighbour (NN) and next-nearest-neighbour (NNN) antiferromagnetic exchange terms $J_{1}$ and $J_{2}$. The chains are coupled along the $c$-axis by $J_{4}$ to form a frustrated two-leg ladder, with an additional weak interladder exchange $J_{3}$. $\mathbf{b}$, Energy-momentum slice of the INS intensity at $T=4 \mathrm{~K}$. Data have been corrected for the isotropic $\mathrm{Cu}^{2+}$ form factor ${ }^{27}$ and intensities placed into absolute units using the incoherent scattering from a vanadium standard. Inset is a schematic illustration of the dispersion of each mode. c. Constant momentum transfer scan at the incommensurate wavevector $Q=(0,0.575,1)$ collected on the SPINS spectrometer at $T=75 \mathrm{mK}$. Solid line is a fit to a resolution-convolved model cross-section and filled areas are contributions from each mode. d, Constant energy transfer cuts, along two-dimensional rods, of scattering at the $k=0.5 \pm 0.075$ positions integrated over $E=2 \pm 0.5 \mathrm{meV}$. Solid lines are the bilayer structure factor as described in the Supplementary Information. Error bars represent one standard deviation.

\section{Valence-bond solid}

The orthorhombic crystal structure of $\mathrm{BiCu}_{2} \mathrm{PO}_{6}$ is shown in Fig. 1a; the structure contains zigzag chains of $\mathrm{Cu}^{2+}$ ions running parallel to the $b$-axis. Magnetic interactions along the chains are frustrated because of a competition between the nearest-neighbour (NN) and next-nearest-neighbour (NNN) antiferromagnetic exchange terms $J_{1}$ and $J_{2}$. The frustrated chains are coupled strongly along the $c$-axis by antiferromagnetic coupling $J_{4}$ to form a two-leg ladder. As a result of the strong antiferromagnetic coupling $J_{4}$, two spins on each rung can form spin-singlets, and the ground state is described as an array of singlets termed a valence-bond solid. The elementary excitations in this case are spin-triplets that can propagate along the chain direction owing to $J_{1}$ and $J_{2}$. Within a single (ladder) bilayer, there are two crystallographically inequivalent copper sites $\left(\mathrm{Cu}_{A}\right.$ and $\mathrm{Cu}_{\mathrm{B}}$ as shown in Fig. 1a). This results in the breaking of inversion symmetry across all magnetic bonds, and consequently anisotropic interactions are permitted in the magnetic Hamiltonian, as will be discussed later.

The dispersion relation, which contains essential information regarding the triplon dynamics such as the effective mass and velocity, is revealed directly by INS measurements. Before discussing our neutron scattering measurements, it is helpful to briefly review the expected excitation spectrum of the frustrated two-leg ladder as realized by $\mathrm{BiCu}_{2} \mathrm{PO}_{6}$, first ignoring any anisotropic interactions. In the strong coupling limit of $J_{1}, J_{2} \ll J_{4}$, the expected excitation spectrum is schematically illustrated in the inset of Fig. $1 b^{10,11}$. The dispersion has a distinct $\mathrm{W}$ shape with a minimum at an incommensurate wavevector; this incommensurate minimum of the gapped spectrum is the manifestation of the magnetic frustration in $\mathrm{BiCu}_{2} \mathrm{PO}_{6}$. As $\mathrm{BiCu}_{2} \mathrm{PO}_{6}$ contains two singlet dimers per unit cell, there are two separate bands of triplons. The bands become degenerate at the point $k=0.5$ and are related by a simple folding of the zone, with the minima of each band appearing at incommensurate wavevectors $k=0.5 \pm \delta$. Anisotropic interactions entering the magnetic Hamiltonian may then further split the degeneracy of each triplon band.

An overview of the zero-field INS measurements is presented in Fig. 1b. The gapped, W-shaped, dispersion of each branch is clearly visible at both $h=0$ and 3 with a bandwidth of $25 \mathrm{meV}$, and incommensurate minima at $k=0.575$ and $k=0.425$. We have not observed any dispersion along the $h$-direction confirming the weak inter-bilayer coupling; however, intensities are strongly modulated with momentum transfers along $h$. This effect is most clearly shown in Fig. 1d, where the intensity along the two-dimensional rods of scattering, at $k=0.5 \pm 0.075$ positions, is plotted. The modulation arises from the interference between the scattering from the two layers within a bilayer, and enables us to probe each mode independently.

What makes $\mathrm{BiCu}_{2} \mathrm{PO}_{6}$ unique among valence-bond solids is the presence of strong anisotropic interactions that qualitatively alter the nature of the triplons. The evidence for anisotropic interactions in $\mathrm{BiCu}_{2} \mathrm{PO}_{6}$ is first borne out by high-resolution measurements around the incommensurate wavevector at $\mathbf{Q}=(0,0.575,1)$ shown in Fig. 1c. These reveal that anisotropies in $\mathrm{BiCu}_{2} \mathrm{PO}_{6}$ completely 


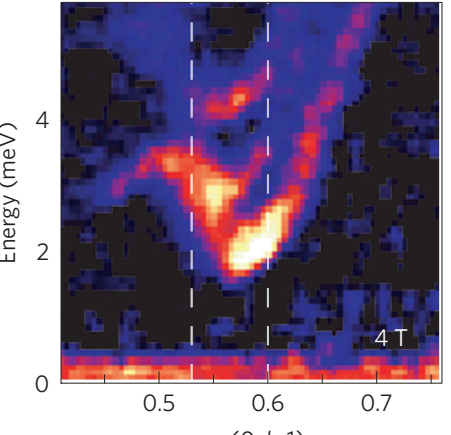

$(0, k, 1)$

d

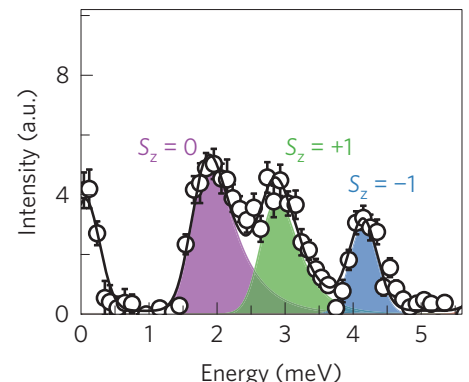

b

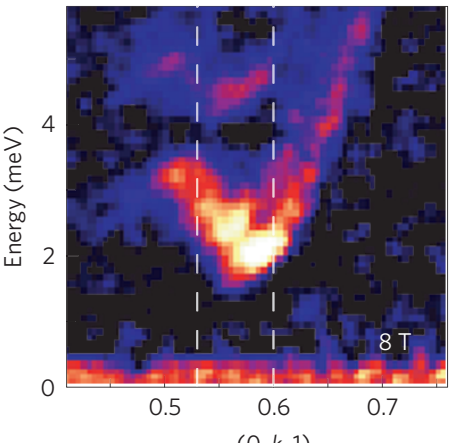

$(0, k, 1)$

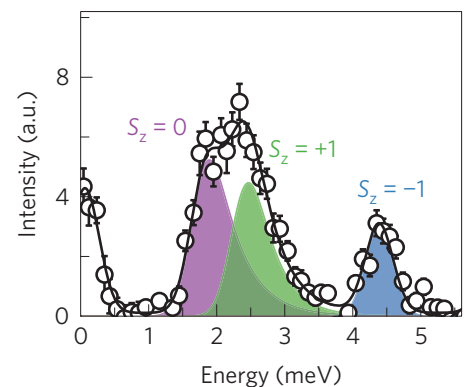

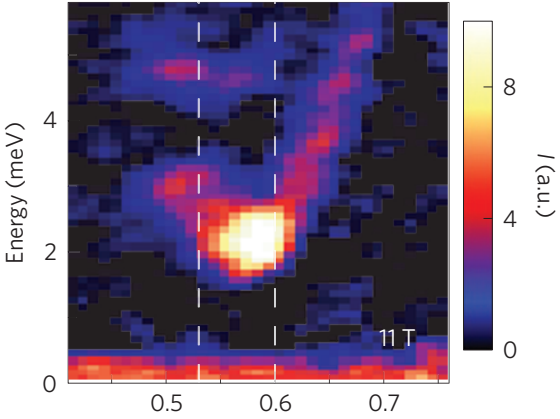

$(0, k, 1)$

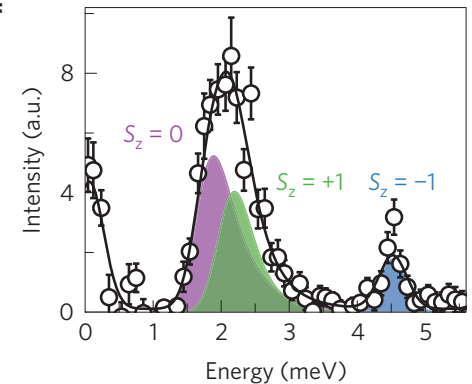

Figure $\mathbf{2}$ | Magnetic field dependence of dynamic magnetic correlations in $\mathbf{B i C u}_{\mathbf{2}} \mathbf{P O}_{\mathbf{6}}$. $\mathbf{a}-\mathbf{c}$, False colour maps of the INS intensity measured on DCS at

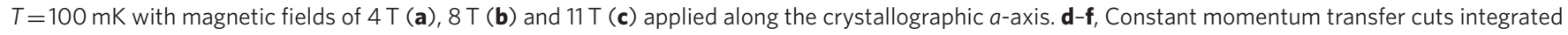
over $k=0.565 \pm 0.035$ r.l.u. and $l=1 \pm 0.05$ r.l.u., integration ranges are represented by dashed white lines in a-c respectively. Solid lines are fit to asymmetric Gaussian functions and filled areas show the contribution from each mode. Asymmetric line shapes result from the steep dispersion and extended momentum integration range. Error bars represent one standard deviation.

split the degeneracy of each primary branch such that three distinct modes are observed. Anisotropy splitting is significant, with the minima of each mode corresponding to gap values of $\Delta_{1}=1.67(2) \mathrm{meV}, \Delta_{2}=2.85(5) \mathrm{meV}$ and $\Delta_{3}=3.90(5) \mathrm{meV}$.

The quantum states of each mode can be further explored through INS measurements performed with applied magnetic fields. The neutron intensities for applied fields of 4,8 and $11 \mathrm{~T}$ are plotted in Fig. 2. No additional splitting of the modes was observed, indicating that anisotropic interactions in the Hamiltonian have completely lifted the $\mathrm{SU}(2)$ spin rotation symmetry. Constant momentum transfer cuts around the incommensurate wavevector, Fig. 2d-f, reveal an anomalous Zeeman behaviour. Rather than splitting into the conventional ordering in energy of $S_{z}=\{+1,0,-1\}$ (refs 6,12), the lowest-energy mode exhibits negligible field dependence and is assigned a $S_{z}=0$ quantum state, whereas the two higher-energy modes have the Zeeman character of $S_{z}=+1$ and $S_{\mathrm{z}}=-1$, respectively; see the field dependence plotted in Fig. 3c. We note that in the presence of anisotropic interactions the singlet and triplet wavefunctions are mixed and $S_{z}$ is no longer a good quantum number. Here we assign each mode a pseudo $S_{z}$ based on on its Zeeman energy, as this provides a convenient labelling scheme.

\section{Non-interacting triplons}

The complete dispersion extracted from INS measurements is plotted in Fig. 3. There are six triplon modes: two primary bands each split into three non-degenerate modes by anisotropic interactions.

To understand the spin dynamics in $\mathrm{BiCu}_{2} \mathrm{PO}_{6}$ we consider the following generic model for interacting spins, $\mathbf{S}_{i}$, on a quasi-2D lattice in the $b-c$ plane

$$
\begin{aligned}
\mathcal{H}= & \sum_{i>j}\left(J_{i j} \mathbf{S}_{i} \cdot \mathbf{S}_{j}+\mathbf{D}_{i j} \cdot \mathbf{S}_{i} \times \mathbf{S}_{j}+\Gamma_{i j}^{\mu \nu} S_{i}^{\mu} S_{j}^{\nu}\right) \\
& -g \mu_{\mathrm{B}} \mathbf{H} \cdot \sum_{i} \mathbf{S}_{i}
\end{aligned}
$$

where relevant Heisenberg exchange terms $J_{i j}$ are shown in Fig. 1a, and $\mathbf{H}$ is an external magnetic field. The model Hamiltonian is an extended $J_{1}-J_{2}-J_{3}-J_{4}$ model including symmetry-allowed anisotropic spin interactions. The antisymmetric DzyaloshinskiiMoriya $\left(\mathbf{D}_{i j}\right)$ and symmetric $\left(\Gamma_{i j}^{\mu \nu}\right)$ anisotropic interactions are constrained by the relation

$$
\Gamma_{i j}^{\mu \nu}=\frac{D_{i j}^{\mu} D_{i j}^{\nu}}{2 J_{i j}}-\frac{\delta^{\mu \nu} \mathbf{D}_{i j}^{2}}{4 J_{i j}}
$$

Both the anisotropic exchange terms originate from spin-orbit coupling ${ }^{13-16}$. Employing a quadratic (non-interacting) bondoperator theory (BOT; refs 17-19) for the valence-bond ordered ground state with valence bonds on $J_{4}$ links, we have found that the INS data is best described with the following coupling constants: $J_{1}=J_{2}=J_{4}=8 \mathrm{meV}, J_{3}=1.6 \mathrm{meV}, D_{1}^{a}=0.6 J_{1}, D_{1}^{b}=0.45 J_{1}$, $\Gamma_{1}^{a a}=0.039 J_{1}, \quad \Gamma_{1}^{b b}=-0.039 J_{1}$ and $\Gamma_{1}^{a b}=\Gamma_{1}^{b a}=0.135 J_{1}$; the calculated triplon dispersion is plotted in Fig. 3a,b. The quadratic BOT captures important details of the low-energy spectra, such as the slight shift of incommensurate minima between each branch, and the overall bandwidth of the excitations. Importantly, this calculation appropriately describes the anomalous Zeeman splitting plotted in Fig. 3c. Furthermore, extending the BOT to determine the field dependence of each mode for fields applied along the $b$ and $c$ directions correctly predicts the hierarchy of critical fields measured previously: $H_{c}^{a}>H_{c}^{b}>H_{c}^{c}$ (ref. 20).

Although the quadratic BOT describes the measured triplon dispersion and the field dependence very well, it is essentially a mean-field expansion and so overestimates the coupling constants. In addition, this quadratic theory fails to capture some very distinct features of the spectrum, including a bending of the triplon modes around $k_{\mathrm{c}} \approx 1 \pm 0.26$ in Fig. $3 \mathrm{a}$ and a excitation continuum resulting from the decay of single triplons in the same region. As we will discuss below, these dynamics ultimately arise as a consequence of the anharmonic magnetic interactions 
a

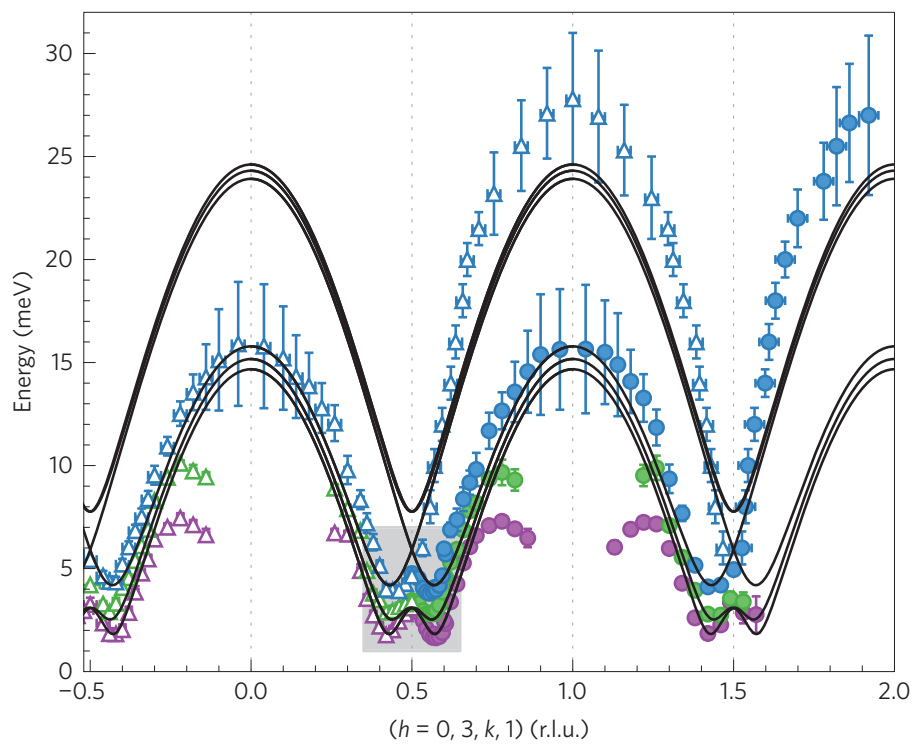

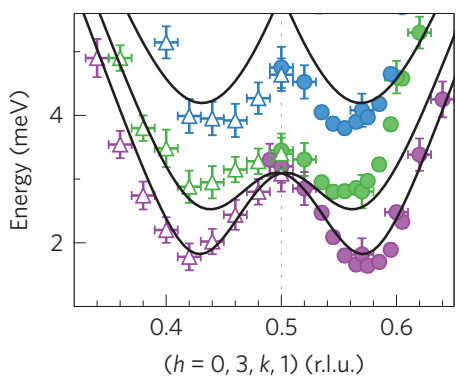

c

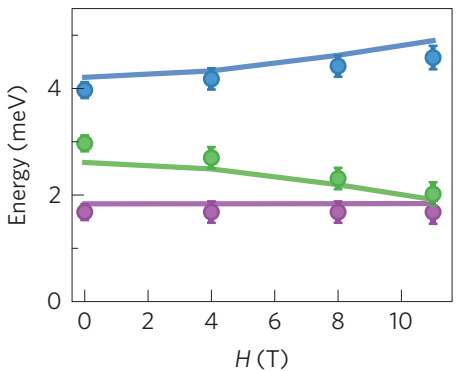

Figure 3 | Triplon dynamics in $\mathrm{BiCu}_{2} \mathrm{PO}_{6} \cdot \mathbf{a}$, Dispersion of triplon excitations in $\mathrm{BiCu}_{2} \mathrm{PO}_{6}$. Points extracted from fits to constant energy transfer and constant momentum transfer scans from SEQUOIA and SPINS data sets. Filled circles correspond to $h=0$, and open triangles correspond to $h=3$; colour coding of symbols indicates the particular triplon band. Solid lines are the dispersion calculated with a quadratic bond-operator theory as described in the text. $\mathbf{b}$, Detailed view around the point $k=0.5 \pm 0.075$, indicated by the black shaded region in $\mathbf{a}$. $\mathbf{c}, \mathrm{Measured}$ energy of each mode at $Q=(0,0.575,1)$ as a function of applied magnetic field. Lines in c are the corresponding calculated Zeeman energies from the bond-operator theory.

which couple single-triplon quasiparticles with a continuum of multi-triplon states.

\section{Multi-particle continuum and level repulsion}

In addition to exciting a single-triplon quasiparticle, a neutron can create two or more triplon excitations simultaneously. For example, a neutron with momentum $\mathbf{Q}$ can create two triplons with momentum $\mathbf{q}$ and $\mathbf{Q}-\mathbf{q}$. These two-triplon excitations form a continuum with a lower bound determined by conservation of momentum and energy $\omega_{2 t}^{\alpha \beta}(\mathbf{Q})=\min _{\mathbf{q}}\left\{\omega^{\alpha}(\mathbf{q})+\omega^{\beta}(\mathbf{Q}-\mathbf{q})\right\}$, where $\omega^{\alpha}(\mathbf{q})$ is the single-triplon dispersion for a band indexed by $\alpha$. In many magnetic materials, the two-triplon (or two-magnon) excitations are usually directly observable only at energies much higher than the one-triplon energy, where the two-triplon density of states becomes large. At low energies the presence of the two-triplon continuum can be revealed through the decay behaviour of a single triplon. In the presence of significant anharmonic (cubic order in bond operators) couplings, the triplon lifetime is significantly reduced, even in the absence of any thermal fluctuations. The decay phenomena manifest in a neutron scattering experiment as a strong damping of the quasiparticle peak and a renormalization of the single-triplon dispersion.

In Fig. $4 \mathrm{~b}$ the quantity $\hbar \omega S(\mathbf{Q}, \omega)$ is plotted to highlight the effects of multi-triplon interactions, including a strongly damped triplon mode at high energies around $k=1$, and the bending and extinction of the lowest triplon modes around $k=0.8$. We note that the observation of triplon decay is possible owing to the crystal structure of $\mathrm{BiCu}_{2} \mathrm{PO}_{6}$, which breaks ladder permutation symmetry explicitly ${ }^{21}$. Another consequence of this low-symmetry structure is that the two-triplon continuum scattering intensity modulates in phase with the single-triplon scattering, preventing us from separating the two, unlike in the case of symmetric ladder compounds ${ }^{22,23}$.

The lower boundaries for two-triplon scattering from each single-triplon branch, $\omega_{2 t}^{\alpha \beta}$, have been determined from the quadratic dispersion (Fig. 3), and are overlaid in Fig. 4a,b. The multi-particle continuum is expected to exist only above this kinematic bound, which also represents the phase space where a quasiparticle decay can occur. To aid a more careful examination of the decay behaviour of three low-energy modes considered in Fig. 2, the momentum-dependent intensity and linewidth of each mode around $k=0.8$ are shown in Fig. $4 \mathrm{c}$,d. The increasing intensity of the highest-energy mode reflects the inclusion of both single- and multi-triplon contributions as the mode smoothly merges with the continuum. Although we observe a continuous increase in the damping of the highest-energy mode, much more marked effects are apparent in the two low-energy triplon branches. The single-triplon dispersions for these branches are strongly renormalized by interactions with the continuum, bending away from the quadratic dispersion. Furthermore, these branches remain resolution-limited in energy at all wavevectors, but terminate abruptly inside the continuum. This is a spectacular example of a spontaneous quasiparticle breakdown, where the decay channels are so effective that an appropriate description of the system in terms of quasiparticles does not exist ${ }^{4,24}$.

To understand this behaviour quantitatively, we have extended the BOT to include anharmonic (both cubic and quartic) interaction terms. In $\mathrm{BiCu}_{2} \mathrm{PO}_{6}$, the Heisenberg exchange terms, $J_{1}, J_{2}$ and $J_{4}$ cancel at cubic order in an interacting bond-operator theory and do not contribute to the spontaneous decay. It is the DzyaloshinskiiMoriya interactions, $D_{1}^{a, b}$, which appear as the strongest anharmonic terms and, thus, are responsible for the spontaneous decay of singleparticle states into multi-particle states. Single-triplon dynamics and decay processes are then identified through the spectral weight function of the triplon Green's function, $A(\mathbf{Q}, \omega)$, shown in Fig. 4a. A region of substantial triplon decay, indicated by a broadening of the spectral function, occurs in an identical region of phase space where triplon decay was observed in the neutron scattering experiments (Fig. 4b). We note that the renormalized coupling constants, $J_{1}=J_{2}=J_{4}=10 \mathrm{meV}, J_{3}=0.2 J_{1}, D_{1}^{a}=D_{1}^{b}=0.3 J_{1}$ and $\Gamma_{1}^{a b}=\Gamma_{1}^{b a}=0.045 J_{1}$, were used to produce the spectral function shown in Fig. 4a. With the inclusion of anharmonic terms, the anisotropic interactions have been reduced from their noninteracting values, illustrating the significance of triplon interaction for describing spin dynamics of $\mathrm{BiCu}_{2} \mathrm{PO}_{6}$. We expect this set of coupling constants to reflect magnetic interactions in $\mathrm{BiCu}_{2} \mathrm{PO}_{6}$ 


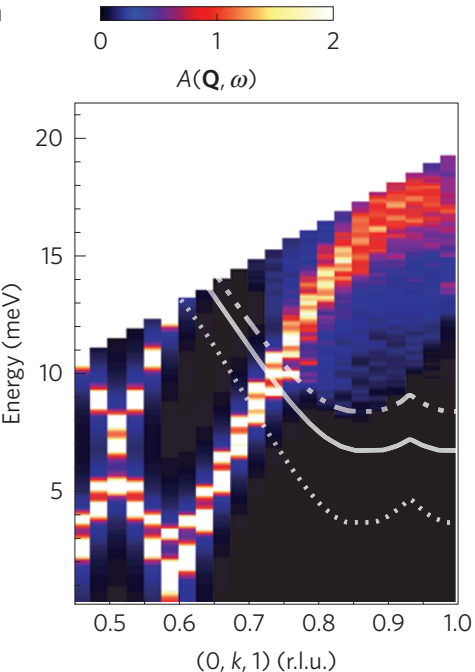

b

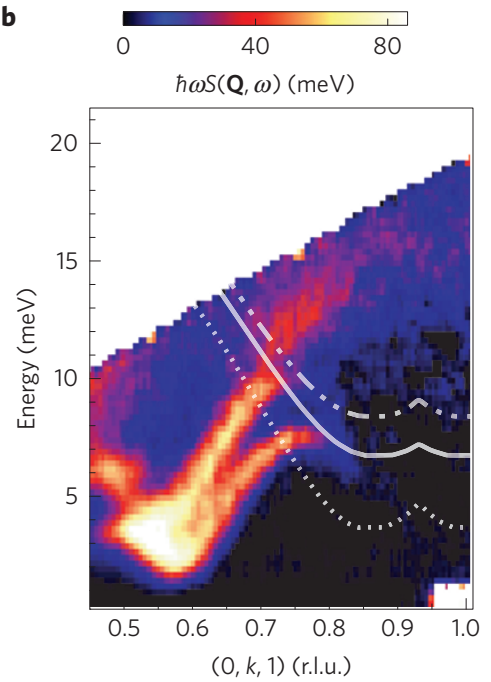

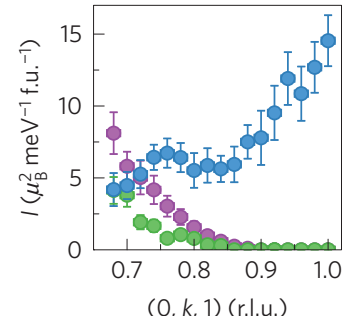

d

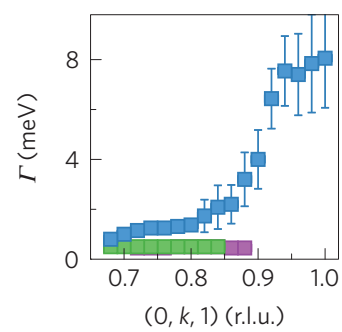

Figure $\mathbf{4}$ | Level repulsion and termination of quasiparticle spectrum in $\mathrm{BiCu}_{2} \mathbf{P O}_{6} . \mathbf{a}$, Spectral function of the triplon $\mathrm{Green}$ 's function, $A(\mathbf{Q}$, $\omega)$, in the interacting triplon theory. $\mathbf{b}, \hbar \omega S(\mathbf{Q}, \omega)$, highlighting the quasiparticle termination and avoided crossing of the triplon branches with the continuum scattering around $Q_{c}$. White lines in $\mathbf{a}$ and $\mathbf{b}$ are the kinematic lower bound for two-triplon scattering from two triplons in the lowest-energy, $\alpha=\beta=1$ (dotted), highest-energy, $\alpha=\beta=3$ (dashed-dot), and $\alpha=2, \beta=3$ (solid) branches. $\mathbf{c}, \mathbf{d}$, Momentum dependence of the spectral weight contained in each branch (c) and the full-width at half-maximum (d); error bars represent one standard deviation. The colour coding is the same as in Fig. 3.

more accurately. Although the basic phenomenology of the triplon decay is well captured by our calculation, the bending of the lowenergy branch is still not accounted for. The bending occurs near the boundary of the two-triplon continuum, possibly due to avoided level crossing between the single quasiparticle and the continuum.

Although detailed observations of triplon dynamics have been made in the past ${ }^{22,25,26}$, clear examples of the spontaneous breakdown of a triplon spectrum as observed here are rare. In earlier studies of organometallic materials, termination of a well-defined triplon peak in the excitation spectrum beyond a critical wavevector was observed ${ }^{3,5}$. The decay process we observe in $\mathrm{BiCu}_{2} \mathrm{PO}_{6}$ is unique, as each triplon branch exhibits different decay behaviour. The highestenergy mode does not bend, but merges smoothly with and decays into the continuum, in contrast to the behaviour of the two lowerenergy branches. As each triplon branch carries a different spin quantum number, the different decay behaviours of each branch might be a direct consequence of spin-dependent selection rules for the quasiparticle decay. Obviously, further theoretical studies are necessary to understand different decay behaviour of triplon branches, and also the strong renormalization of the single-triplon branch observed here. In addition, unlike organometallic materials investigated in earlier studies, $\mathrm{BiCu}_{2} \mathrm{PO}_{6}$ is an inorganic compound in which magnetic coupling parameters can be tuned more easily. Further investigation of doped $\mathrm{BiCu}_{2} \mathrm{PO}_{6}$ could also be useful for understanding the triplon decay in this material.

In summary, we have mapped the quasiparticle excitation spectra in the quantum magnet $\mathrm{BiCu}_{2} \mathrm{PO}_{6}$ through comprehensive INS measurements. The overall triplon dispersion is captured by a quadratic bond-operator theory for the valence-bond solid and we find that large anisotropic interactions are necessary to describe the excitation spectrum. These anisotropic couplings appear as anharmonic, non-particle conserving, terms in the bond-operator Hamiltonian and facilitates strong interaction between the triplon quasiparticles. Strong hybridization between the lowest-triplon branches and multi-triplon continuum scattering in the neighbourhood of the critical wavevector results in a renormalization of single-triplon dispersion indicative of avoided level crossing. Perhaps the most important feature of the excitation spectrum in $\mathrm{BiCu}_{2} \mathrm{PO}_{6}$ is this selective hybridization, renormalization, and termination of the two lowest branches, distinct from the smooth merging of the highest-energy branch into a continuum. Further theoretical investigation of interacting triplons could shed light on the origin of the observed unusual decay behaviour.

\section{Methods}

Methods and any associated references are available in the online version of the paper.

Received 27 May 2015; accepted 14 October 2015; published online 30 November 2015

\section{References}

1. von Neumann, J. \& Wigner, W. P. Über merkwüdige diskrete Eigenwerte. Phys. Z. 30, 465-467 (1929).

2. Hopfield, J. J. Theory of the contribution of excitons to the complex dielectric constant of crystals. Phys. Rev. 112, 1555-1567 (1958).

3. Stone, M. B., Zaliznyak, I. A., Hong, T., Broholm, C. L. \& Reich, D. H. Quasiparticle breakdown in a quantum spin liquid. Nature 440 , 187-190 (2006)

4. Zhitomirsky, M. E. \& Chernyshev, A. L. Colloquium: Spontaneous magnon decays. Rev. Mod. Phys. 85, 219-242 (2013)

5. Masuda, T. et al. Dynamics of composite Haldane spin chains in IPA- $\mathrm{CuCl}_{3}$. Phys. Rev. Lett. 96, 047210 (2006).

6. Giamarchi, T., Rüegg, C. \& Tchernyshyov, O. Bose-Einstein condensation in magnetic insulators. Nature Phys. 4, 198-204 (2008).

7. Okolowicz, J., Ploszajczak, M. \& Rotter, I. Dynamics of quantum systems embedded in a continuum. Phys. Rep. 374, 271-383 (2003).

8. Miroshnichenko, A. E., Flach, S. \& Kivshar, Y. S. Fano resonances in nanoscale structures. Rev. Mod. Phys. 82, 2257-2298 (2010).

9. Yoon, Y. et al. Coupling quantum states through a continuum: A mesoscopic multistate Fano resonance. Phys. Rev. X 2, 021003 (2012).

10. Tsirlin, A. A. et al. Bridging frustrated-spin-chain and spin-ladder physics: Quasi-one-dimensional magnetism of $\mathrm{BiCu}_{2} \mathrm{PO}_{6}$. Phys. Rev. B 82, 144426 (2010)

11. Lavarélo, A., Roux, G. \& Laflorencie, N. Melting of a frustration-induced dimer crystal and incommensurability in the $J_{1}-J_{2}$ two-leg ladder. Phys. Rev. B 84, 144407 (2011).

12. Matsumoto, M., Normand, B., Rice, T. M. \& Sigrist, M. Magnon dispersion in the field-induced magnetically ordered phase of $\mathrm{TlCuCl}_{3}$. Phys. Rev. Lett. 89, 077203 (2002)

13. Dzyaloshinsky, I. A thermodynamic theory of weak ferromagnetism of antiferromagnetics. J. Phys. Chem. Solids 4, 241 (1958). 
14. Moriya, T. Anisotropic superexchange interaction and weak ferromagnetism. Phys. Rev. 120, 91-98 (1960).

15. Shekhtman, L., Entin-Wohlman, O. \& Aharony, A. Moriya's anisotropic superexchange interaction, frustration, and Dzyaloshinsky's weak ferromagnetism. Phys. Rev. Lett. 69, 836-839 (1992).

16. Yildirim, T., Harris, A. B., Aharony, A. \& Entin-Wohlman, O. Anisotropic spin Hamiltonians due to spin-orbit and Coulomb exchange interactions. Phys. Rev. B 52, 10239-10267 (1995).

17. Sachdev, S. \& Bhatt, R. N. Bond-operator representation of quantum spins: Mean-field theory of frustrated quantum Heisenberg antiferromagnets. Phys. Rev. B 41, 9323-9329 (1990).

18. Gopalan, S., Rice, T. M. \& Sigrist, M. Spin ladders with spin gaps: A description of a class of cuprates. Phys. Rev. B 49, 8901-8910 (1994).

19. Matsumoto, M., Normand, B., Rice, T. M. \& Sigrist, M. Field- and pressure-induced magnetic quantum phase transitions in $\mathrm{TlCuCl}_{3}$. Phys. Rev. $B$ 69, 054423 (2004).

20. Kohama, Y. et al. Anisotropic cascade of field-induced phase transitions in the frustrated spin-ladder system $\mathrm{BiCu}_{2} \mathrm{PO}_{6}$. Phys. Rev. Lett. 109, 167204 (2012).

21. Schmidt, K. P. \& Uhrig, G. S. Spectral properties of magnetic excitations in cuprate two-leg ladder systems. Mod. Phys. Lett. B 19, 1179-1205 (2005).

22. Notbohm, S. et al. One- and two-triplon spectra of a cuprate ladder. Phys. Rev. Lett. 98, 027403 (2007)

23. Schmidiger, D. et al. Spectral and thermodynamic properties of a strong-leg quantum spin ladder. Phys. Rev. Lett. 108, 167201 (2012).

24. Zhitomirsky, M. E. Decay of quasiparticles in quantum spin liquids. Phys. Rev B 73, 100404 (2006).

25. Xu, G., Broholm, C., Reich, D. H. \& Adams, M. A. Triplet waves in a quantum spin liquid. Phys. Rev. Lett. 84, 4465-4468 (2000).
26. Stone, M. B., Zaliznyak, I., Reich, D. H. \& Broholm, C. Frustration-induced two-dimensional quantum disordered phase in piperazinium hexachlorodicuprate. Phys. Rev. B 64, 144405 (2001).

27. Brown, P. J. International Tables for Crystallography Vol. C, Ch. 4.4.5, 454-461 (Springer, 2006).

\section{Acknowledgements}

We would also like to thank G. Uhrig, O. Tchernyshyov and S. K. Kim for helpful discussions. This research was supported by NSERC of Canada, Canada Foundation for innovation, Canada Research Chairs Program, and Centre for Quantum Materials at the University of Toronto. Work at ORNL was sponsored by the Division of Scientific User Facilities, Office of Basic Energy Science, US Department of Energy (DOE). Work at NIST utilized facilities supported in part by the National Science Foundation under Agreement No. DMR-0944772.

\section{Author contributions}

K.W.P. and Y.-J.K. conceived the experiments. K.W.P., Y.Q., L.W.H. G.E.G. and A.I.K. performed the experiments and K.W.P. analysed the data. C.R. provided additional data K.H. and Y.B.K. developed the theoretical model and performed calculations. G.J.S. and F.C.C. provided the sample. K.W.P. and Y.-J.K. wrote the paper with contributions from all co-authors.

\section{Additional information}

Supplementary information is available in the online version of the paper. Reprints and permissions information is available online at www.nature.com/reprints.

Correspondence and requests for materials should be addressed to Y.-J.K.

\section{Competing financial interests}

The authors declare no competing financial interests. 


\section{Methods}

All measurements used the same $4.5 \mathrm{~g}$ single crystal as previous studies ${ }^{28}$. Magnetic excitations in $\mathrm{BiCu}_{2} \mathrm{PO}_{6}$ were mapped through inelastic neutron scattering (INS) measurements performed on a number of instruments. High-energy time-of-flight neutron scattering measurements where carried out on the SEQUOIA spectrometer at the Spallation Neutron Source (SNS), covering the full dynamic range of excitations in $\mathrm{BiCu}_{2} \mathrm{PO}_{6}$ with a fine energy resolution of $\Delta E \sim 0.8 \mathrm{meV}$ at the elastic line. Measurements on SEQUOIA were performed with a fixed incident neutron energy of $E_{\mathrm{i}}=40 \mathrm{meV}$ and the fine-resolution Fermi-chopper $\left(\mathrm{FC}_{2} ;\right.$ ref. 29) rotating at $360 \mathrm{~Hz}$. The sample was mounted with the $(h, k, 0)$ plane lying in the horizontal scattering plane of the instrument and $(h, 0,0)$ initially aligned along the incident neutron wavevector $\mathbf{k}_{\mathbf{i}}$. To map the complete dynamic structure factor $S(\mathbf{Q}, \omega)$ the sample was rotated through $180^{\circ}$ in $0.5^{\circ}$ steps. All measurements on SEQUOIA were performed with the sample held at a temperature of $4 \mathrm{~K}$. Another set of high-resolution measurements where conducted on the SPINS cold triple-axis spectrometer at the NIST Center for Neutron Research (NCNR). Here the sample was mounted in the $(0, k, l)$ scattering plane and all measurements used a fixed final energy of $E_{\mathrm{f}}=3.7 \mathrm{meV}$ employing a vertically focusing PG monochromator, a flat PG analyser, and a $\mathrm{BeO}$ filter between the sample and analyser. The spectrometer collimation sequence was Guide- $80^{\prime}-80^{\prime}$-Open, resulting in an energy resolution of $\Delta E \sim 0.1 \mathrm{meV}$ at the elastic line. For the duration of the experiment, the sample was mounted on a $\mathrm{Cu}$ mount and temperature was controlled in a ${ }^{3} \mathrm{He}$ dilution refrigerator. Measurements in an applied magnetic field were carried out on the DCS time-of-flight spectrometer at NIST (ref. 30). All measurements on DCS were performed using a fixed incident neutron wavelength of $\lambda_{\mathrm{i}}=2.9 \AA$. The energy resolution on DCS was $\Delta E \sim 0.3 \mathrm{meV}$ at the elastic line. The sample was mounted in the $(0, k, l)$ scattering plane with $(0,0, l)$ initially at $50^{\circ}$ from the incident neutron beam and then rotated through $120^{\circ}$ in $0.5^{\circ}$ steps throughout the measurement. The sample was fixed on a Cu mount in a $11.5 \mathrm{~T}$ vertical field cryomagnet with a dilution refrigerator insert. A magnetic field between 4 and $11.5 \mathrm{~T}$ was applied along the $a$-axis and the sample was held at $T=100 \mathrm{mK}$ for the duration of the measurements. Because of the very narrow magnet aperture, measurements with applied field on DCS were confined to the $(0, k, l)$ scattering plane, with momentum transfers in the vertical direction limited to $h=0 \pm 0.2$ r.l.u.

\section{References}

28. Plumb, K. W. et al. Incommensurate dynamic correlations in the quasi-two-dimensional spin liquid $\mathrm{BiCu}_{2} \mathrm{PO}_{6}$. Phys. Rev. B 88, 024402 (2013).

29. Granroth, G. E. et al. SEQUOIA: A newly operating chopper spectrometer at the SNS. J. Phys. Conf. Ser. 251, 012058 (2010).

30. Copley, J. R. D. \& Cook, J. C. The disk chopper spectrometer at NIST: A new instrument for quasielastic neutron scattering studies. Chem. Phys. 292, 477-485 (2003). 